ARTIGO

do $10.22481 /$ praxisedu.v15i33.5283

\title{
LENGUAJE Y ESTRATEGIAS UTILIZADOS POR FUTUROS PROFESORES DE EDUCACIÓN PRIMARIA EN LA RESOLUCIÓN DE PROBLEMAS COMBINATORIOS
}

\author{
LANGUAGE AND STRATEGIES USED BY PROSPECTIVE PRIMARY EDUCATION \\ TEACHERS IN SOLVING COMBINATORIAL PROBLEMS
}

\section{LINGUAGEM E ESTRATÉGIAS UTILIZADAS PELOS FUTUROS PROFESSORES DE EDUCAÇÃO PRIMÁRIA NA RESOLUÇÃO DE PROBLEMAS COMBINATÓRIOS}

\author{
María M. Gea \\ Universidad de Granada - España \\ Carmen Batanero \\ Universidad de Granada - España
}

Alex Venegas

Colegio Santa María Eufrasia - Chile

\begin{abstract}
Resumen: En este estudio exploratorio se analiza la influencia del tipo de lenguaje y las estrategias utilizadas en la resolución sobre la corrección de la solución de tres problemas de combinaciones por parte de 62 profesores de Educación Primaria en formación. Asimismo se estudia la diferencia de lenguaje y estrategia dependiendo del modelo combinatorio (partición, colocación y selección). Se obtiene un porcentaje de respuestas correctas similar al obtenido por Navarro-Pelayo con estudiantes de 14 años sin instrucción en los problemas cuyo modelo combinatorio es la selección o colocación e igual al de los estudiantes de 17 años con instrucción en el modelo de partición. El lenguaje predominante es el verbal, y simbólico y la estrategia más frecuente es la enumeración, generalmente sistemática, así como la fijación de variables. La corrección de la respuesta no depende del lenguaje utilizado, pero si de la estrategia utilizada, apareciendo diferencias del lenguaje y estrategia según el modelo combinatorio del enunciado.
\end{abstract}

Palabras clave: Futuros profesores; Problemas combinatorios; Lenguaje y estrategias.

Abstract: In this exploratory study the influence of the type of language and strategies used in the
resolution of three problems of combinations by 62 prospective Primary Education teachers on the
correction of the solution is analysed. Likewise, the difference of language and strategy depending on
the combinatorial model (partition, placement and selection) is studied. The percentage of correct
answers is similar to that obtained by Navarro-Pelayo with 14 -year-old students without instruction in
the selection or placement problems, and equal to that of the 17 -year-old students with instruction in
the partition model. The predominant language is verbal and symbolic and the most frequent strategy 
is enumeration, generally systematic, as well as fixing variables. The correction of the answer does not depend on the language used, but on the strategy, appearing language and strategy differences according to the combinatorial model of the problem.

Keywords: Prospective teachers; Combinatorial problems; Language and strategies.

Resumo: Neste estudo exploratório é analisada a influência do tipo de linguagem e das estratégias utilizadas na resolução sobre a correção da solução de três problemas de combinações por 62 professores do Ensino Fundamental em formação Da mesma forma, a diferença de linguagem e estratégia é estudada dependendo do modelo combinatório (partição, posicionamento e seleção). Uma porcentagem de acertos é semelhante à obtido por Navarro-Pelayo com alunos de 14 anos sem instrução nos problemas cujo modelo combinatório é a seleção ou colocação e igual àquele dos alunos de 17 anos com instrução no modelo de partição. A linguagem predominante é verbal e simbólico e a estratégia mais frequente é a enumeração, geralmente sistemática, bem como a fixação de variáveis. A correção da resposta não depende da linguagem utilizada, mas da estratégia utilizada, surgindo diferenças de linguagem e estratégia de acordo com o modelo combinatório do problema.

Palabras clave: Pofessores en formaçao; Problemas combinatorios; Linguagen e estratégias.

\section{Introducción}

La Combinatoria es hoy día una rama de las matemáticas con múltiples aplicaciones, por ser la base de la matemática discreta. Aunque su principal interés dentro del currículo escolar es el campo de la probabilidad, mediante ella se ejercitan otros contenidos matemáticos, como el recuento, cálculo, la clasificación, relación de equivalencia y de orden, conjuntos, aplicaciones y funciones (English, 2005). Está también relacionada con el desarrollo cognitivo de conceptos como la disyunción, correspondencia, asociación, implicación o exclusión y de procesos como la generalización y el razonamiento sistemático (Inhelder y Piaget, 1958). Por otro lado, es un campo muy adecuado para la práctica de resolución de problemas, ya que permite plantear problemas sencillos, contextualizados en actividades cotidianas, en los que se puede poner en práctica diferentes estrategias de resolución (Lockwood, 2013). Según Pessoa y Borba (2010, p. 2):

[...] el raciocinio combinatorio como un tipo de pensamiento que implica conteo, pero que va más allá de la enumeración de elementos de un conjunto. En la Combinatoria se cuentan, basándose en el raciocinio multiplicativo, grupos de posibilidades, a través de una acción sistemática, sea por el uso de fórmula, sea por el desarrollo de una estrategia que dé cuenta de atender a los requisitos de esos tipos de problemas, como la constitución de agrupaciones, la determinación de posibilidades y su recuento.

Aunque formalmente no se incluye explícitamente la combinatoria en el currículo 
español de Educación Primaria (MECD, 2014), si la podemos encontrar en el estudio de la probabilidad elemental. Además, en este currículo aparece un bloque de contenidos transversal sobre resolución de problemas, que incluye los siguientes temas, que se pueden ejercitar mediante el trabajo combinatorio (p. 19388):

Planificación del proceso de resolución de problemas: Análisis y comprensión del enunciado. Estrategias y procedimientos puestos en práctica: hacer un dibujo, una tabla, un esquema de la situación, ensayo y error razonado, operaciones matemáticas adecuadas, etc.

A pesar de su sencillez conceptual, la investigación previa, ha descrito dificultades en la resolución de problemas combinatorios en estudiantes de diversas edades (por ejemplo, en Batanero, Navarro-Pelayo y Godino, 1997 o Jalan, Nusantara, Subanji y Chandra, 2016). No obstante, Fischbein (1975) sugirió que la enseñanza del razonamiento combinatorio es posible a partir de los 8-10 años, con la ayuda del diagrama en árbol y recursos manipulativos y que, en ausencia de una enseñanza específica, muchas personas no llegan a desarrollar completamente dicha capacidad. Siguiendo sus sugerencias, diversos autores describen experiencias de evaluación o desarrollo del razonamiento combinatorio de los niños (English, 1999; 2005; Pessoa y Borba, 2009, 2010).

Pero una enseñanza efectiva requiere la preparación adecuada de los profesores, basada en una evaluación previa de sus necesidades formativas. Con el fin de contribuir a esta necesidad, el objetivo de esta investigación fue realizar una evaluación del lenguaje matemático y las estrategias utilizadas en la resolución de problemas elementales de combinatoria en los estudiantes españoles que se preparan como futuros profesores de Educación Primaria. Adicionalmente se tratará de analizar la posible influencia del lenguaje y las estrategias en la corrección de los problemas planteados y si difieren según el modelo combinatorio del enunciado del problema (teniendo en cuenta los modelos descritos por Dubois, 1984) que se describen a continuación.

\section{Fundamentos}

Nos basamos, en primer lugar en los modelos combinatorios y estrategias en la resolución de problemas combinatorios definidos por varios autores y por otro lado en las investigaciones previas; todo ello se resume a continuación. 


\subsection{Marco teórico}

En nuestro trabajo tendremos en cuenta el modelo combinatorio implícito, una variable del enunciado de los problemas que fue identificada por Dubois (1984). Esta variable se basa en la modelización que puede realizarse del contexto de los enunciados de los problemas y es relevante desde el punto de vista matemático, pues el tipo de objetos implicados en la resolución de cada problema varía según el tipo de modelo combinatorio. El autor considera tres tipos básicos de modelo combinatorio:

- Modelo de selección: Este tipo de problemas está centrado en la idea de muestreo de una población de m elementos, de la cual se eligen $n$; el muestreo puede ser con reemplazamiento (y da lugar a las operaciones combinatorias con repetición) o sin él (operaciones ordinarias); si la muestra elegida es ordenada, se trata de variaciones y si no lo es de combinaciones. Este es el modelo que se usa para definir las operaciones combinatorias en España y un ejemplo es el Problema 2 planteado en nuestro estudio.

- Modelo de colocación: Este tipo de problemas implica la colocación de un conjunto de $\mathrm{m}$ elementos en otro conjunto de $\mathrm{n}$ celdas. Ahora se presentan muchas más posibilidades, pues cada uno de los dos conjuntos puede estar ordenado o no y se puede decidir que en las celdas vayan uno o más elementos. Matemáticamente es isomorfo al conjunto de posibles aplicaciones entre dos conjuntos de elementos, y no existe un isomorfismo completo entre el modelo de colocación y el de selección. Un ejemplo es nuestro Problema 1.

- Modelo de partición: Se centra en la división de un conjunto de m elementos en n subconjuntos excluyentes; tanto el conjunto original como las partes pueden estar o no ordenadas; las partes pueden tener igual o diferente número de elementos (ver Problema 3). Además, existe una correspondencia biunívoca entre los modelos de colocación y partición, debido a que la partición de un conjunto de m elementos en $n$ subconjuntos puede traducirse como la colocación de m elementos en n casillas.

Lockwood (2013) sugiere que los estudiantes razonan sobre los problemas combinatorios de acuerdo a un modelo que incluye tres componentes: a) las expresiones matemáticas o fórmulas que reconocen y tratan de aplicar al problema, como la fórmula de combinaciones o simplemente la regla del producto; b) Los procesos de enumeración, que consisten en una serie de pasos que realizan o imaginan para completar la tarea combinatoria 
y c) Los conjuntos de elementos que deben ser combinados o mezclados. Estos tres componentes deben interactuar convenientemente entre sí, para asegurar el éxito en la tarea. Además, en nuestro trabajo tendremos en cuenta las estrategias de resolución de problemas combinatorios descritas por Roa (2000), la mayoría de las cuales son comunes a cualquier proceso de resolución de problemas:

- Traducción del problema a otro más simple o conocido. Fue utilizada en la resolución de problemas combinatorios en la investigación de Roa, Batanero, Godino y Cañizares (1997) y puede llevarse a cabo mediante una disminución de subconjuntos, o elementos del problema, para, una vez resuelto, generalizar la solución al problema inicial. Esta estrategia no apareció en ninguno de los participantes de nuestro estudio.

- Fijación de variables. Esta estrategia es muy utilizada como se muestra, por ejemplo en Roa (2000). Consiste en mantener de manera constante una o más variables involucradas en el problema, para de este modo disminuir su complejidad. Por ejemplo, en el problema 2 se puede suponer que la maestra elige en primer lugar a Elisa y resolver el problema con esta restricción. A continuación se generaliza la solución para el problema inicial.

- Descomposición del problema en partes. Se trata de buscar una disminución de la dificultad del problema inicial, que se divide en subproblemas cuyas soluciones individuales, combinadas, permiten obtener la solución del problema inicial. Puede incluir a las anteriores.

- Principio aditivo o regla de la suma. Este principio se aplica en la gran mayoría de los problemas combinatorios, bien solo o combinada con otras estrategias. Implica formar subconjuntos excluyentes de cierta cantidad de elementos con las características identificadas en el enunciado del problema, de modo que su unión abarca el total del conjunto deseado.

- Principio multiplicativo o regla del producto. Esta estrategia es necesaria en los problemas que incluyan dos o más subconjuntos dependientes, o en dos situaciones donde la primera dependa de la segunda y consiste en multiplicar el número de elementos de diferentes conjuntos para hallar el del espacio producto (Roa, 2000). Por ejemplo, en un grupo de 5 alumnos hay 3 mujeres y 2 hombres. ¿Cuántas parejas (de diferente sexo) pueden formarse en este grupo? A diferencia de la regla de la suma, se debe tener en cuenta que las decisiones de escoger un hombre y una mujer son 
situaciones dependientes, por lo que la solución del problema es el producto del número de elementos de cada decisión.

- Uso de fórmulas combinatorias. Muchos estudiantes intentan resolver los problemas combinatorios mediante la aplicación de fórmulas, según la operación combinatoria a la que se refiera el problema, lo cual requiere identificar correctamente la operación combinatoria requerida y los parámetros que deben utilizar (Batanero, Godino y Navarro- Pelayo, 1997).

- Estrategia de enumeración. Este tipo de estrategia es utilizada en muchas investigaciones y se refiere al listado, sistemático o no sistemático, al inventariar todas las posibles opciones (o al menos una cantidad suficiente para poder generalizar a la solución del problema), y con ello cuantificar para solucionar el problema. Este tipo de estrategia suele venir acompañada del uso de lenguaje gráfico y simbólico, que facilita la enumeración de los posibles resultados, principalmente en las primeras edades (Batanero, Godino y Navarro-Pelayo, 1994, English, 1999, 2005).

Finalmente utilizaremos el constructo conflicto semiótico que ofrece el enfoque ontosemiótico (Godino, Batanero y Font, 2007; 2019) para evidenciar las relaciones entre los objetos matemáticos y los procesos interpretativos en las prácticas matemáticas y que se relaciona con la función semiótica. Los autores asumen que los objetos matemáticos surgen de las prácticas realizadas para resolver los problemas que les dan sentido y, dada la diversidad de objetos matemáticos, se producen una serie de procesos interpretativos durante dichas prácticas, por medio de funciones semióticas. Cualquier tipo de objeto (concepto, proposición, lenguaje, procedimiento, problema) puede ser la expresión o el contenido de una función semiótica y el tipo de funciones semióticas utilizadas en la resolución de problemas, puede aumentar o disminuir la dificultad de la tarea propuesta (Godino, 2002).

En consecuencia, las actividades de interpretación o de proposición de funciones semióticas que desarrolle el sujeto serán consideradas correctas, cuando éstas sean conformes con las propuestas otorgadas por la institución. En caso contrario, encontramos un conflicto semiótico o: "Disparidad o discordancia entre los significados atribuidos a una expresión por dos sujetos (personas o instituciones) en interacción comunicativa, que puede explicar las dificultades y limitaciones de los aprendizajes y las enseñanzas implementadas" (GODINO, 2002, p.250). 


\subsection{ANTECEDENTES}

Los precursores en el estudio del razonamiento combinatorio fueron Inhelder y Piaget (1958), quienes llevaron a cabo investigaciones con niños de diferentes edades y concluyeron que el desarrollo de la capacidad de razonamiento combinatorio es característico de la edad de las operaciones formales (a partir de 12-13 años) y se desarrolla conjuntamente con la lógica proposicional.

Fischbein (1975), por su parte, consideró que dicho razonamiento no se adquiere espontáneamente, sino que se requiere la instrucción específica, con ayuda de material manipulativo y diagrama del árbol. Llevó a cabo experimentos con niños a partir de los 10 años para probar su teoría. Fischbein consideró el diagrama del árbol un modelo facilitador para la generalización constructiva, permitiendo desarrollar el pensamiento recursivo, típico de la combinatoria.

English (1999) realizó un estudio con 96 niños entre 7 y 12 años a los que propone problemas combinatorios relacionados con la regla del producto de orden 2 (combinar dos conjuntos de elementos) y 3 (tres tipos de elementos), estudiando las estrategias de solución. Sugiere que la experiencia con los problemas de orden 2 llevó a los niños a adaptarlas para los de orden 3. Generalmente se usó la enumeración, muchas veces en forma no sistemática. El $83 \%$ de los niños de 12 años resolvió todos los problemas de orden 2 y el $64 \%$ los de orden 3.

Nuestro antecedente más importante es Navarro-Pelayo (1994), quien evaluó el razonamiento combinatorio de estudiantes de secundaria mediante un cuestionario con tareas basadas en diferentes operaciones combinatorias y en los tres modelos combinatorios de Dubois (1984), dirigido a dos muestras de estudiantes de secundaria con y sin instrucción previa en el tema (720 en total). Determina el siguiente orden de dificultad de las operaciones combinatorias (de más sencillo a más difícil): Permutaciones, combinaciones, variaciones, permutaciones con repetición y variaciones con repetición.

Identifica diferentes tipos de error en la resolución de los problemas y obtiene en general peores resultados en los estudiantes sin instrucción y en los problemas de variaciones con repetición. En nuestro trabajo utilizamos directamente tres de los problemas planteados por dicha autora, uno de cada uno de los modelos combinatorios de colocación, selección y partición, e informaremos con más detalles de los resultados de la autora en estos problemas al comentar los nuestros. Utilizamos únicamente la operación combinatoria de combinación, la segunda más sencilla en el trabajo de Navarro-Pelayo, pues ella no usó todos los modelos 
combinatorios para la operación de permutaciones. En Batanero, Navarro-Pelayo y Godino (1997) se describen los errores más frecuentemente encontrados en su muestra, algunos de los cuáles, que interpretamos en términos de conflictos semióticos, aparecen también en nuestro trabajo.

Otro antecedente es Roa (2000), quien aplica el cuestionario de Navarro Pelayo a 90 estudiantes del último curso de la licenciatura de matemáticas. Aunque los resultados son bastante mejores que los de la autora, todavía encuentra muchas dificultades, especialmente en el modelo combinatorio de partición. El autor se centra preferentemente en analizar las estrategias utilizadas, que hemos descrito en la sección anterior e indica que las estrategias generales de resolución de problemas (traducir el problema, fijar variables, descomponer en partes) son las que llevan a mejores soluciones de los problemas.

\section{METODOLOGÍA}

La muestra considerada estuvo formada por un grupo de 62 estudiantes de segundo curso en el Grado de Magisterio de Educación Primaria, en Granada, que realizaron el año anterior otra asignatura obligatoria de matemáticas, La tasa de aprobados de la asignatura de matemáticas en la muestra fue del $60 \%$, siendo sus notas de acceso a la universidad en su mayoría notable (7-8 puntos sobre 10). Por tanto, los estudiantes tenían una formación suficiente para abordar los problemas, pues además, habían estudiado combinatoria, dentro del tema de probabilidad, en varios cursos de la educación secundaria.

La recogida de datos se realizó como parte de la asignatura sobre Enseñanza y Aprendizaje de las Matemáticas, que cursaban estos alumnos, cuya parte práctica se organiza en talleres donde el grupo se divide en otros pequeños (20-25 estudiantes) en sesiones de una hora de duración. Los datos se recogieron en uno de estos talleres, dirigido a reforzar su conocimiento sobre resolución de problemas y estrategias de resolución. A estos estudiantes se propuso los tres problemas mostrados en el Cuadro 1, todos ellos tomados de la investigación de Navarro-Pelayo (2014), a los cuáles respondieron individualmente y por escrito, dedicándose posteriormente otra sesión a la discusión colectiva de las soluciones y corrección de posibles errores. 


\section{Cuadro 1. Cuestionario propuesto a los estudiantes}

Problema 1. Disponemos de tres cartas iguales. Deseamos colocarlas en cuatro sobres de diferentes colores: amarillo, blanco, crema y dorado. Si cada sobre sólo puede contener, a lo sumo, una carta. ¿De cuántas formas podemos colocar las tres cartas en los cuatro sobres diferentes? Ejemplo: podemos colocar una carta en el sobre amarillo, otra en el blanco y otra en el crema.

Problema 2. Una maestra tiene que elegir tres estudiantes para borrar la pizarra. Para ello dispone de cinco voluntarios: Elisa, Fernando, Germán, Jorge y María. ¿De cuántas formas puede elegir tres de estos alumnos? Ejemplo: Elisa, Fernando y María.

Problema 3. María y Carmen tienen cuatro cromos numerados de 1 a 4 . Deciden repartírselos entre las dos (dos cromos para cada una). ¿De cuántas formas se pueden repartir los cromos? Ejemplo: María puede quedarse con los cromos 1 y 2 , y Carmen con los cromos 3 y 4.

Fuente: Elaborado por los autores

Como se indicó anteriormente, para el cuestionario se han elegido tres problemas que corresponden a la operación combinatoria de combinación, uno de cada uno de los tres modelos combinatorios considerados por Dubois (1984). El primero de ellos sería un problema del modelo de colocación, pues se dispone de dos conjuntos de objetos (cartas y sobres), debiéndose colocar los primeros en los segundos. La condición es que las cartas son indistinguibles y sólo se puede incluir una carta en cada sobre. Puesto que el estudiante sólo ha estudiado el modelo de selección, ha de realizar una traducción del problema a dicho modelo. Para resolverlo, también se puede aplicar la fórmula de combinación de $n=4$ sobres, tomados en grupos de $k=3$ (considerando que se deben elegir tres de los cuatro sobres para colocar las cartas y que no importa el orden de elección): $C_{k}^{n}=4$. Igualmente se podrían enumerar todas las combinaciones posibles mediante lenguaje simbólico, indicando con letras el color de cada sobre donde se introduce la carta y sabiendo que siempre quedará un sobre vacío en cada combinación: $\{\mathrm{ABC} ; \mathrm{ABD} ; \mathrm{ACD} ; \mathrm{BCD}\}$.

En el segundo problema, el modelo combinatorio es el de selección, por lo que no se precisa traducir el enunciado. También se puede resolver mediante la fórmula, donde se selecciona $k=3$ personas de $n=5$ personas (total del grupo), sin necesidad de considerar el orden: $C_{3}^{5}=10$. Si identificamos los estudiantes por sus iniciales, podemos establecer la enumeración del total de posibles combinaciones de 5 estudiantes tomados de 3 en 3.

El tercer problema corresponde a un modelo de partición, pues se trata de partir un conjunto de objetos (los cuatro cromos) en dos partes con igual número de cromos. El enunciado se debe traducir a un modelo de selección, considerando que dar dos cromos a una de las niñas es lo mismo que seleccionar dos cromos para ella y el resto se le da a la otra niña. 
Puesto que el orden en que se reciben los cromos no importa, se resuelve con la operación combinatoria de combinación, según la fórmula pertinente: $C_{2}^{4}=6$.También se puede utilizar la enumeración, posiblemente con ayuda de una tabla donde se representen los nombres de las chicas ( $\mathrm{M}=$ Maria y $\mathrm{C}=\mathrm{Carmen})$ y los cromos para cada una de ellas, mostrando así la relación de este tipo de problemas con los de colocación.

\section{RESULTADOS}

A continuación se presentan los porcentajes de respuestas correctas, parcialmente correctas e incorrectas a los problemas, seguido por un análisis del lenguaje utilizado y de las estrategias de resolución.

\subsection{CORRECCIÓN DE LA SOLUCIÓN}

En la Tabla 1 presentamos los resultados respecto a la corrección de la solución aportada por los estudiantes de nuestra muestra, comparándolos con los del trabajo de Navarro-Pelayo (1994) en los mismos problemas resueltos por estudiantes de Bachillerato, con o sin instrucción previa en el tema. La autora solo consideró respuestas completamente correctas, considerando las parcialmente correctas como incorrectas. En nuestro caso hemos considerado que la respuesta es parcialmente correcta cuando se ha identificado correctamente los datos e iniciado el proceso de solución de forma adecuada y sistemática, pero la generalización errónea provoca que sus resultados sean incorrectos.

Los resultados en todos los problemas son mejores en nuestra investigación, en comparación con la de Navarro-Pelayo (1994) excepto en el Problema 2, donde sus estudiantes con instrucción obtienen mejores resultados. En nuestro caso no se obtienen respuestas en blanco.

Tabla 1. Porcentaje de respuestas según grado de corrección en los problemas

\begin{tabular}{|c|c|c|c|c|c|c|c|c|c|}
\hline & \multirow{2}{*}{\multicolumn{3}{|c|}{$\begin{array}{c}\text { Futuros profesores } \\
(\mathrm{n}=62)\end{array}$}} & \multicolumn{6}{|c|}{ Alumnos de Bachillerato (Navarro-Pelayo, 1994) } \\
\hline & & & & \multicolumn{3}{|c|}{ Con instrucción $(\mathrm{n}=352)$} & \multicolumn{3}{|c|}{ Sin instrucción $(n=368)$} \\
\hline & $\mathrm{C}$ & $\mathrm{PC}$ & I & $\mathrm{C}$ & I & NR & $\mathrm{C}$ & I & NR \\
\hline Problema 1: Colo & 48,4 & 19,4 & 32,2 & 26,7 & 57,1 & 16,2 & 26,9 & 53,3 & 19,8 \\
\hline Problema 2. Selección & 25,8 & 40,3 & 33,9 & 46 & 47,7 & 6,3 & 22,6 & 72 & 5,4 \\
\hline Problema 3. Partición & 58,1 & 33,8 & 8,1 & 37,2 & 56 & 6,8 & 31 & 60,6 & 8,4 \\
\hline
\end{tabular}

$\mathrm{C}=$ Correcta, $\mathrm{PC}=$ Parcialmente correcta, $\mathrm{I}=$ Incorrecta, $\mathrm{NR}=$ No responde

Fuente: Elaborado por los autores 
La autora concluyó que los problemas correspondientes al modelo de selección eran los más simples de resolver por los participantes en su trabajo y, la dificultad en los problemas de partición no disminuyó después de la instrucción. En nuestra investigación, el problema correspondiente al modelo de selección fue el más difícil, siendo el más sencillo el problema de partición. La autora no analizó las estrategias utilizadas por sus estudiantes y los nuestros son de mayor edad, por lo que estos factores podrían explicar las diferencias encontradas.

\subsection{LENGUAJE UTILIZADO}

El lenguaje matemático se considera la parte ostensiva de las prácticas matemáticas en el EOS, que le atribuye un doble carácter, representacional y operativo (Godino, Batanero y Font, 2007). En consecuencia, nos hemos interesado por analizar el tipo de lenguaje matemático que utilizan los futuros profesores para responder al problema, variable no analizada en las investigaciones previas. A continuación indicamos las categorías utilizadas para clasificar el lenguaje, presentando ejemplos de las soluciones en el Problema 1.

Lenguaje tabular: El lenguaje tabular tiene un uso muy extenso en matemáticas, aunque su simplicidad es sólo aparente, debido a la variedad que se pueden construir. Duval (2002) señala dos aspectos básicos de las mismas, que son, la organización de la propia representación, es decir la componente semiótica asociada a la tabla, y la componente cognitiva, que requiere la comprensión de la información representada en ella. Su estructura que permite ordenar la información por medio de la intersección de filas y columnas, distribuyéndolas visualmente, lo que la convierte en una disposición gráfica en la que se muestra información cualitativa y/o cuantitativa (Gabucio, Martí, Enfedaque, Gilabert, y Konstantinidou, 2010).

En la solución de los problemas planteados algunos estudiantes utilizan tablas para representar la colocación de las cartas en los sobres, diferenciando el conjunto de cartas en un eje y en otro el de sobres y marcando en las celdas de la tabla las posibilidades, es decir como ayuda a la enumeración (ver ejemplo en la Figura 1, donde el estudiante por este medio llega a una solución correcta. De forma similar se utiliza en el problema de partición. 


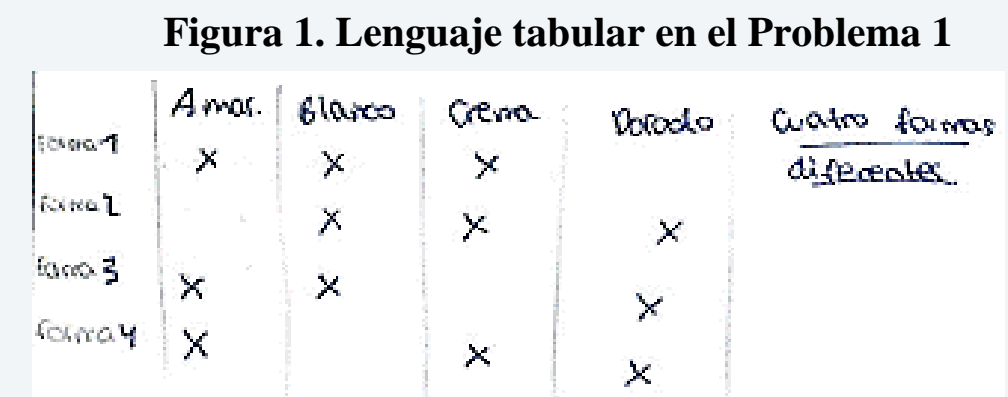

Fuente: Respuesta del estudiante TC

Lenguaje Simbólico: Las notaciones simbólicas y expresiones algebraicas permiten una comunicación comprimida entre individuos, pudiendo trabajar a un alto nivel de complejidad y actualmente se considera la invención de sus propios símbolos por los estudiantes como una parte relevante de su actividad matemática (Gravemeijer, Lehrer, van Oers y Verschaffel, 2013). En nuestra investigación consideramos este tipo de lenguaje cuando los participantes representan los elementos del conjunto analizado o los grupos formados mediante letras para facilitar sus procedimientos de enumeración y también cuando se emplean símbolos algebraicos en las fórmulas combinatorias.

En la Figura 2 observamos un ejemplo en que el estudiante asocia a cada sobre a la letra inicial de su color (A, B, C, D) y a cada carta un número para resolver el Problema 1, aunque no completa correctamente la enumeración.

Figura 2. Lenguajes simbólico e icónico en el problema 1

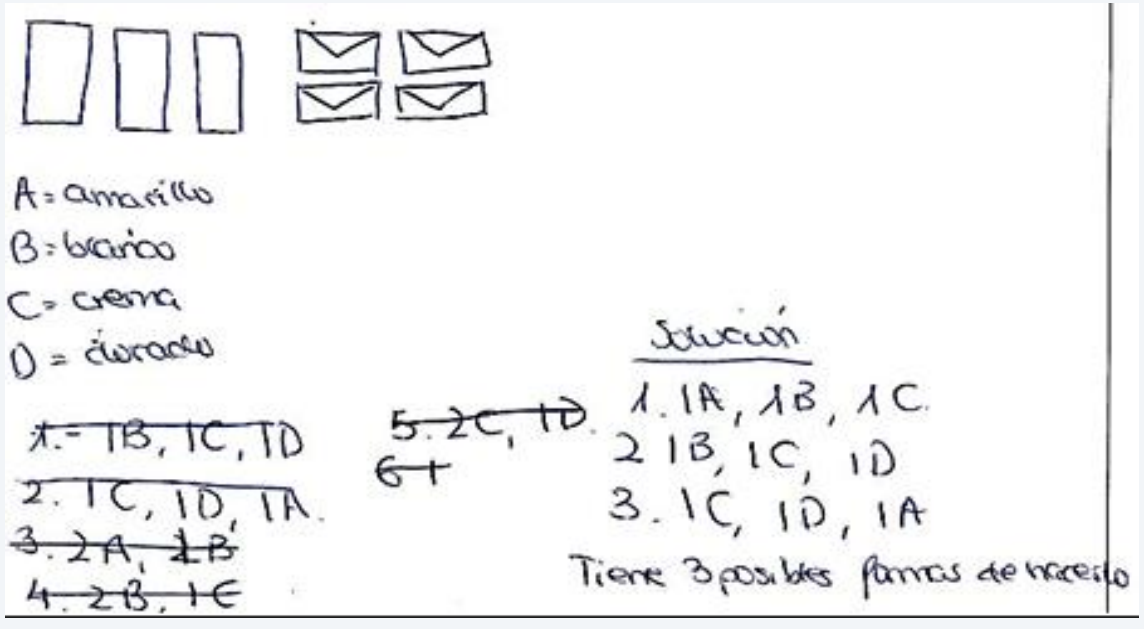

Fuente: Respuesta del estudiante JG

Lenguaje Gráfico: Los participantes utilizan gráficos, generalmente el diagrama del árbol como ayuda a la enumeración y generalización. Fischbein (1975) consideró el diagrama en árbol como una recurso productivo y útil en la visualización de la estructura de la solución de los problemas combinatorios $\mathrm{y}$, además, dota de sentido intuitivo a las operaciones combinatorias. Un ejemplo en el Problema 1 se muestra en la Figura 3, donde RO escribe 
todas las posibilidades mediante un diagrama del árbol y luego justifica verbalmente cuales de estos casos considera para responder al problema (Figura 3).

\section{Figura 3. Lenguajes gráfico y verbal en el problema 1}

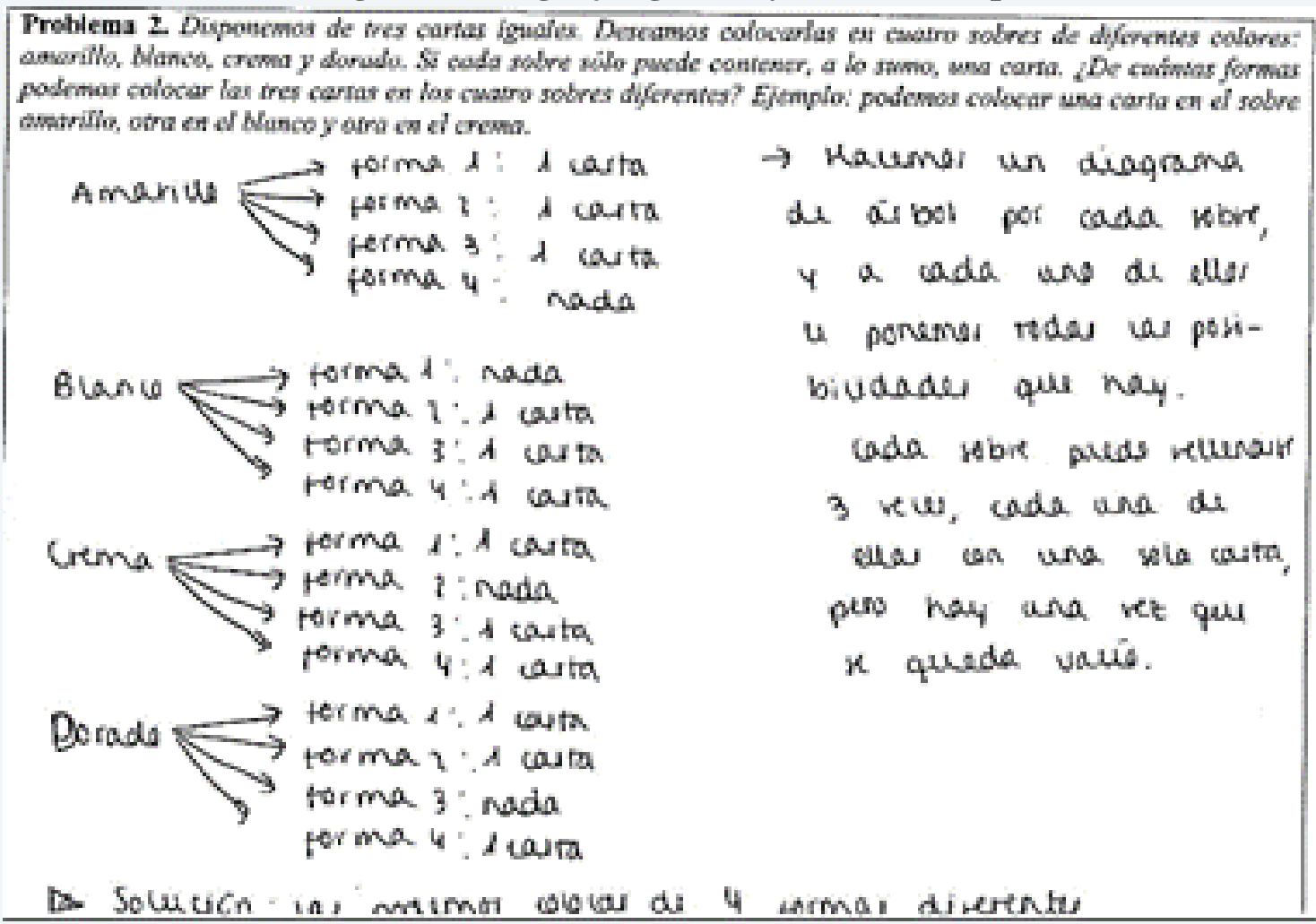

Fuente: Respuesta del estudiante RO

Lenguaje Icónico: Algunos participantes utilizan este tipo de lenguaje representando con dibujos que aluden a los elementos que deben ser combinados. Por ejemplo, el mismo estudiante JG, que había usado el lenguaje simbólico, lo complementa dibujando los sobres y las cartas para comprender mejor el enunciado del problema 1 (Figura 2).

Lenguaje verbal: El lenguaje verbal es también parte de la actividad matemática, donde se mezclan palabras específicas con otras utilizadas en la vida diaria, siendo las primeras mucho más precisas (Schleppegrell, 2007). En nuestro estudio son muchos los futuros profesores que explica verbalmente el procedimiento de resolución, por lo que este es el lenguaje más utilizado en las respuestas, bien sólo o combinado con otros tipos de lenguaje. Por ejemplo, en la Figura 3 se combina con el lenguaje gráfico. Observamos en el ejemplo que se mezclan expresiones cotidianas con otras de significado matemático como diagrama en árbol y posibilidades. 


\subsubsection{Influencia del lenguaje en la corrección de la respuesta}

Una vez descritos los tipos de lenguaje considerado presentamos el estudio estadístico de su uso por parte de los sujetos de la muestra. En primer lugar se ha analizado la posible influencia que el tipo de lenguaje utilizado tenga sobre la corrección de la respuesta (Tabla 1). Aunque el menor porcentaje de respuestas correctas se asocia al lenguaje simbólico, si consideramos las respuestas parcialmente correctas, los peores resultados están asociados al lenguaje tabular, que es el que produce mayor número de respuestas incorrectas, debido a las dificultades de los estudiantes en la construcción de las tablas. Este resultado confirma que la simplicidad del lenguaje tabular es sólo aparente (Gabucio et al., 2010). No obstante, las diferencias de respuestas correctas, parcialmente correctas e incorrectas, según el lenguaje no llegan a ser estadísticamente significativas en el test Chi cuadrado de homogeneidad de proporciones de los tres tipos de respuesta según lenguaje.

Tabla 1. Porcentaje de tipo de respuesta según lenguaje utilizado

\begin{tabular}{cccc}
\hline & \multicolumn{3}{c}{ Tipo de respuesta } \\
\cline { 2 - 4 } Lenguaje & Correcta & $\begin{array}{c}\text { Parcialmente } \\
\text { correcta }\end{array}$ & Incorrecta \\
\hline Tabular & 46,7 & 13,3 & 40,0 \\
Simbólico & 35,3 & 33,3 & 31,4 \\
Gráfico & 40,4 & 34,0 & 25,5 \\
Icónico & 46,4 & 28,6 & 25,0 \\
Verbal & 45,8 & 31,3 & 22,9 \\
\hline
\end{tabular}

\subsubsection{Lenguaje utilizado en los diferentes problemas}

Para estudiar si el lenguaje depende del modelo combinatorio del enunciado, en la Tabla 2 mostramos el porcentaje de estudiantes que usa diferente tipo de lenguaje en los tres problemas. Aunque los lenguajes verbal y simbólico son siempre los más frecuentes, el porcentaje de uso de estos dos tipos de lenguaje es mayor en el segundo problema (problema de selección). Se utiliza más el lenguaje simbólico en el segundo problema, de selección, que es el estudiado por los alumnos y donde han reconocido con más facilidad la operación combinatorias de combinaciones. El lenguaje gráfico, generalmente el diagrama en árbol tiene mayor uso en el tercero (partición), donde pocos estudiantes reconocieron las combinaciones y lo utilizaron como ayuda a la enumeración. Finalmente el lenguaje icónico es más frecuente en los problemas 1 y 3 (colocación y partición). Al analizar la posible asociación entre estas 
dos variables, el test Chi cuadrado dio resultados estadísticamente significativos $(\mathrm{Chi}=28.5$, g.l.=8, p.0004), lo que sugiere un diferente uso del lenguaje e función del modelo combinatorio del enunciado.

Tabla 2. Porcentaje de tipo de lenguaje utilizado en cada problema

\begin{tabular}{lccc}
\hline & \multicolumn{3}{c}{ Problema } \\
\cline { 2 - 4 } Lenguaje & P1. Colocación & P2. Selección & P3. Partición \\
\hline Verbal & 38,8 & 46,2 & 33,0 \\
Simbólico & 25,0 & 38,5 & 28,7 \\
Gráfico & 9,5 & 10,6 & 20,9 \\
Tabular & 11,2 & 1,9 & 6,1 \\
Icónico & 15,5 & 2,9 & 11,3 \\
\hline
\end{tabular}

Fuente: Elaborado por los autores

\subsection{ESTRATEGIAS DE RESOLUCIÓN}

En esta sección se presenta el análisis de las estrategias utilizadas por los futuros profesores para resolver los problemas, comenzando por la descripción de ejemplos de las mismas, seguidas de su análisis estadístico.

Enumeración sistemática: La enumeración o listado de las posibilidades es una estrategia muy utilizada en la resolución de problemas combinatorios, cuando una primera lectura del enunciado no lleva directamente a la identificación de la operación combinatoria (Batanero et al., 1994). Se encuentra incluso en investigaciones con niños, como la de English (1999, 2005). Consideramos que la enumeración es sistemática, cuando se caracteriza por un sistema, generalmente fijar un elemento, producir un subconjunto de posibilidades mediante la enumeración de una parte de la solución y seguir fijando otros elementos para repetir el proceso. Este tipo de estrategia, bien aplicada, conduce a la respuesta correcta, ya que se enumeran el total de combinaciones posibles (MELUSOVA; VIDERMANOVA, 2015). Como ejemplo, en la Figura 3 se muestra la enumeración sistemática de RO para resolver el problema 1.

En algunas respuestas esta estrategia se utilizó incorrectamente, produciendo un exceso o defecto de posibilidades. Así, en la Figura 2, se mostró cómo JG obtiene por defecto tres de las cuatro combinaciones posibles, mediante una enumeración sistemática; mientras que AM (Figura 4), obtiene un número mayor, debido a que comete un error al considerar distinguibles 
las cartas (que son idénticas según el enunciado del problema). La confusión entre objetos distinguibles e indistinguibles se trataría de un conflicto semiótico, pues se confunde una propiedad (ser iguales) de los objetos a combinar con otra (ser diferentes) y fue considerado simplemente como un error por Navarro-Pelayo (1994).

\section{Figura 4. Enumeración sistemática incorrecta en el Problema 1}

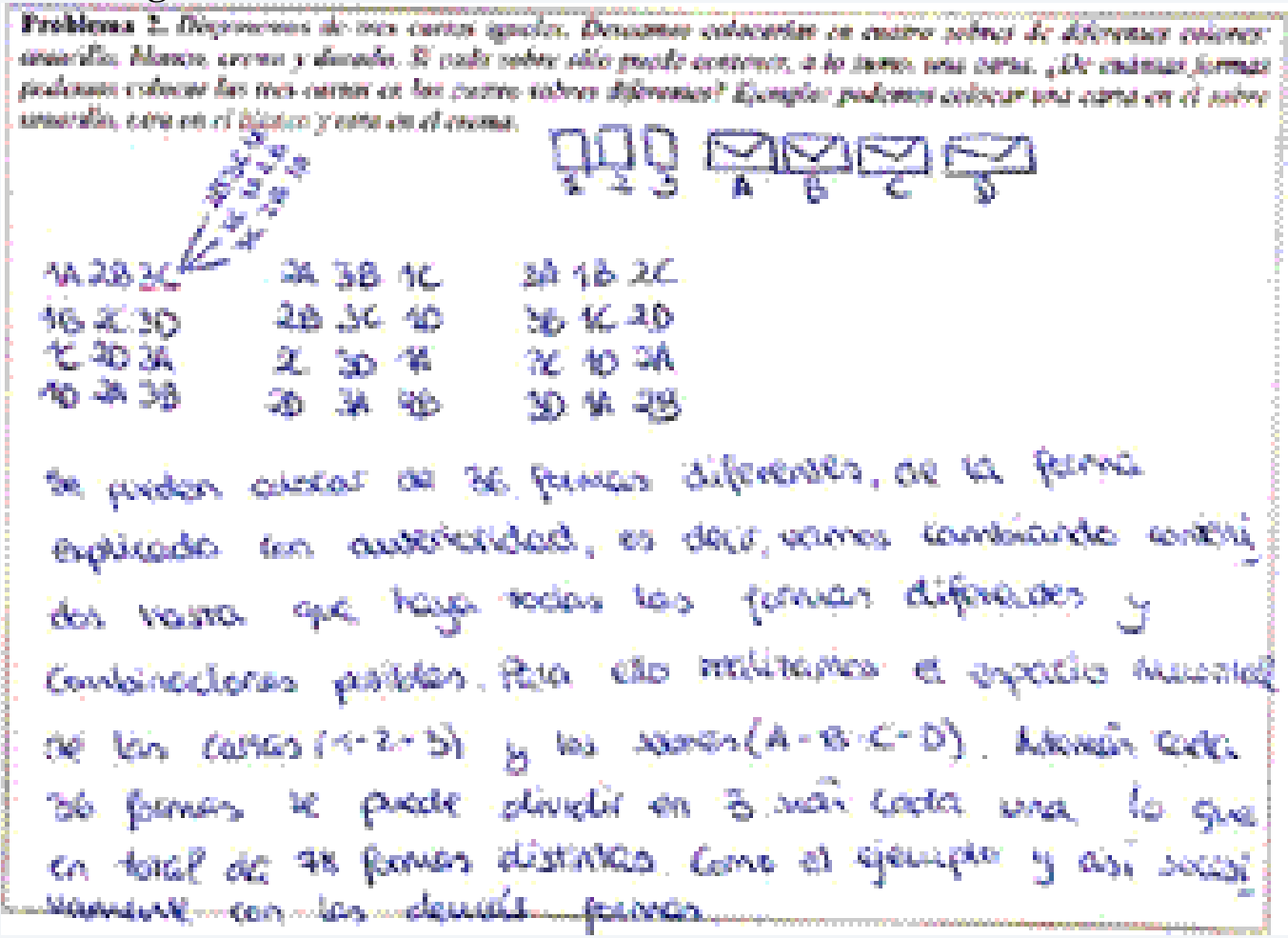

Fuente: Respuesta del estudiante AM

Enumeración no sistemática: Algunos futuros profesores utilizan la enumeración como forma de resolver el problema, pero no establecen un orden sistemático al enumerar las combinaciones, por lo que, en su mayoría obtienen respuestas parcialmente correctas o incorrectas. Este tipo de enumeración es típica de niños que no han alcanzado suficiente nivel de razonamiento combinatorio (English, 1999, 2005), pero aun así aparece en nuestra muestra. Vemos un ejemplo en la Figura 3 donde EB no llega a construir todos los casos posibles en el problema 2 .

Figura 5. Enumeración no sistemática en el Problema 2

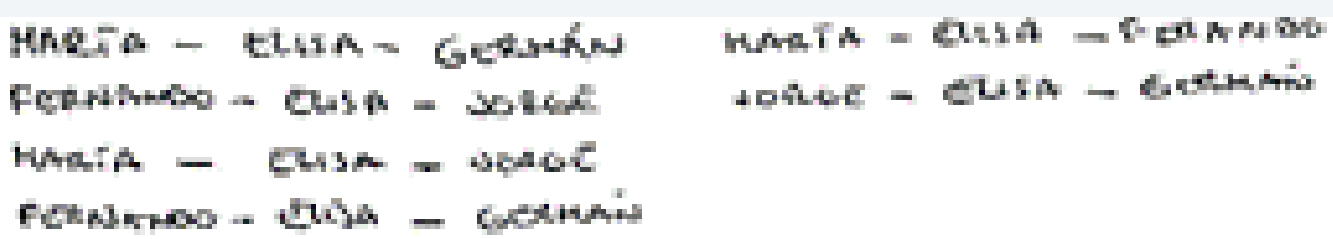

Fuente: Respuesta del estudiante EB 
Principio multiplicativo: Algunos futuros profesores aplicaron el principio multiplicativo, para obtener el número de elementos de una combinación como producto del número de elementos de los conjuntos que intervienen en el problema. Esta aplicación no siempre se realiza correctamente como se muestra en la Figura 6, donde, para resolver el problema 1, el estudiante NR multiplica el número de sobres (4), por el de cartas (3), estableciendo como resultado un total de 12 posibilidades. De nuevo se observa en este ejemplo la confusión entre elementos distinguibles e indistinguibles identificada por NavarroPelayo (2004), que nosotros hemos interpretado como conflicto semiótico.

\section{Figura 6. Principio multiplicativo en el problema 1}

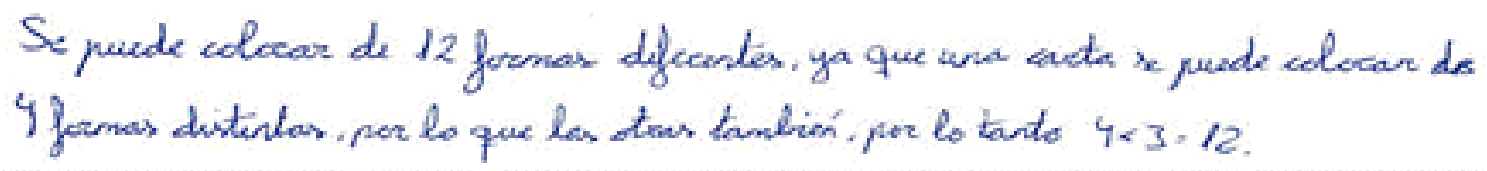

Fuente: Respuesta del estudiante NR

Fijación de variables y descomposición del problema: Esta estrategia fue utilizada por gran parte de los estudiantes al igual que en la investigación de Roa (2000). La fijación de variables en muchos casos viene acompañada generalmente de una descomposición del problema en partes, para resolver cada parte por separado y finalmente hallar la solución global. Así, por ejemplo en la Figura 7, LI para resolver el Problema 2 combina la fijación de variables y descomposición del problema. El estudiante analiza por separado las posibilidades de cada estudiante, para posteriormente, eliminar aquellas repetidas, no obstante, repitió una de ellas, obteniendo más combinaciones de las necesarias.

Figura 7. Descomposición del problema 2

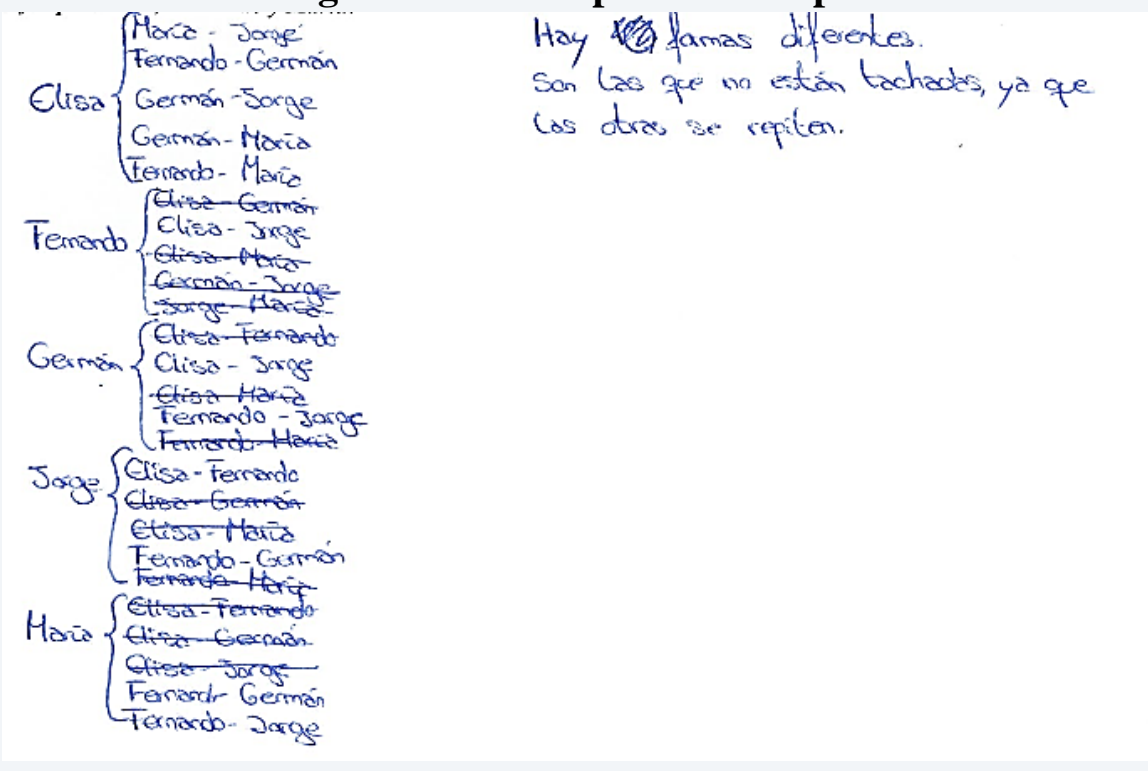

Fuente: Respuesta del estudiante LI 
Principio aditivo: En algunos casos se utiliza la suma del cardinal de los conjuntos de posibles soluciones al establecer el número de combinaciones. Por ejemplo, en el problema 1 SH describe su razonamiento para establecer las 4 combinaciones, según esta estrategia. Pensando la situación de que un sobre se quede vacío. Así, se suma el total de posibilidades en cada caso, que es: $1+1+1+1=4$ (Figura 8$)$.

Figura 8. Principio aditivo en el Problema 1

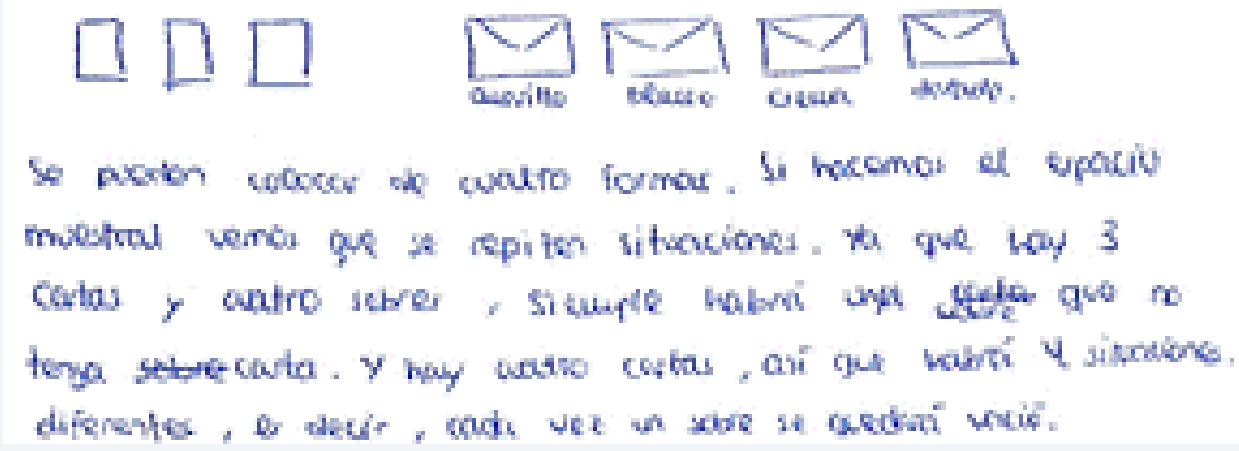

Fuente: Respuesta del estudiante SH

Otras: Otras estrategias se basan en el uso de tablas o de fórmulas combinatorias, generalmente mal aplicadas, como ocurre con BM en el Problema 1 (Figura 9), donde aplica incorrectamente la fórmula de las variaciones con repetición. Observamos un conflicto semiótico en la confusión del tipo de operación combinatoria, identificado también por Navarro-Pelayo (2004). También se incluye en esta categoría las estrategias confusas o poco explicadas.

\section{Figura 9. Fórmula incorrectamente aplicada en el problema 1}

Para sílo una carto, existin 4 formas diferentis de ponerla en un scbre.

Sin embarg, siblo son 3 cavias enternces mulliplicames

Las posibritidedes par ell mímero de cartas: $3^{3}$

$3 \times 3 \times 3=27$

Son 27 formas distintas de celocas las cartas.

Fuente: Respuesta del estudiante BM 


\subsubsection{Influencia de la estrategia en la corrección de la respuesta}

Una vez presentadas las categorías del análisis de estrategias, detallamos el análisis estadístico de las mismas. En la Tabla 3 se presentan los porcentajes de respuestas correctas, parcialmente correctas e incorrectas según estrategia empleada en el conjunto de problemas, con el fin de analizar la influencia de la estrategia en la corrección de la solución. Observamos que el mayor número de respuestas correctas está ligado a la enumeración sistemática y en segundo lugar al hecho de fijar variables. Por otro lado el principio multiplicativo, al ser aplicado incorrectamente llevó generalmente a respuestas incorrectas y con menor frecuencia la enumeración no sistemática y otras estrategias, como uso de tablas o fórmulas combinatorias o el principio aditivo. Al realizar el contraste Chi-cuadrado para analizar la posible asociación entre estrategia y tipo de respuesta se obtuvo un resultado estadísticamente muy significativo $(\mathrm{Chi}=53.6798$, g.1. $=4, \mathrm{p}<0.00001)$.

Tabla 3. Porcentaje de tipo de respuesta según estrategia utilizada

\begin{tabular}{lccc}
\hline \multirow{2}{*}{ Estrategia } & \multicolumn{3}{c}{ Tipo de respuesta } \\
\cline { 2 - 4 } & Correcta & Parcialmente correcta & Incorrecta \\
\hline Enumeración sistemática & 54,2 & 37,5 & 8,3 \\
Enumeración no sistemática & 33,3 & 26,7 & 40,0 \\
Fija variables y descompone & 45,6 & 41,2 & 13,2 \\
Principio multiplicativo & 3,7 & 29,6 & 66,7 \\
Otras & 34,7 & 22,4 & 42,9 \\
\hline
\end{tabular}

Fuente: Elaborada por los autores

\subsubsection{Estrategia utilizada en los diferentes problemas}

Otra cuestión de interés es analizar si las estrategias utilizadas están asociadas al tipo de modelo combinatorio en el enunciado del problema. Para estudiar este punto, en la Tabla 4 se presentan los porcentajes de estrategias utilizadas en cada uno de los problemas.

Tabla 4. Porcentaje de estrategias utilizadas en cada problema.

\begin{tabular}{lrrr}
\hline & \multicolumn{3}{c}{ Problema } \\
\cline { 2 - 4 } Estrategia & P1. Colocación & P2. Selección & P3. Partición \\
\hline Enumeración sistemática & 16,4 & 30,0 & 49,3 \\
Enumeración no sistemática & 26,0 & 40,0 & 19,4 \\
Fija variables y descompone & 4,1 & 4,4 & 11,9 \\
Otras & 19,2 & 10,0 & 4,5 \\
P. multiplicativo & 34,2 & 15,6 & 14,9 \\
\hline
\end{tabular}


Observamos que la enumeración sistemática es más frecuente en el problema de partición, siendo la no sistemática de mayor frecuencia en los otros problemas. El principio multiplicativo se asocia principalmente al problema de colocación, donde los estudiantes trataron (con poco éxito en general) de obtener una fórmula, al no identificar la operación combinatoria. Se utiliza poco la descomposición del problema y fijación de variables, siendo estas estrategias generales de resolución de problemas, ya que los estudiantes se han centrado en estrategias típicamente combinatorias. Al realizar el contraste Chi-cuadrado para analizar la posible asociación entre estrategia y tipo de respuesta se obtuvo un resultado estadísticamente muy significativo (Chi=37,6415, g.1.=4, p < 0.00001).

\subsection{Conflictos semióticos en la resolución de la tarea}

El análisis de las soluciones a las tareas ayudó a identificar algunos conflictos semióticos (Godino, Batanero y Font, 2007; 2019), donde encontramos diferencia en los procesos interpretativos que en su actividad matemática realizan los estudiantes, en relación con los considerados correctos desde el punto de vista institucional.

En primer lugar aparecen conflictos en la interpretación del enunciado del problema, que listamos a continuación:

- C1: Confusión entre el tipo de objetos que se debe combinar (distinguibles o indistinguibles). Este conflicto aparece en el Problema 1, donde consideraron las cartas distinguibles, al igual que ocurrió en la investigación en el 32,6\% de los estudiantes de Navarro-Pelayo (1994),

- C2: Confundir conjuntos ordenados y no ordenados, por ejemplo, considerar el orden de los sobres (que no influye en la solución) en el Problema 1, que también apareció en Navarro-Pelayo (1994), donde el 51,2\% de participantes con instrucción trataron de aplicar la fórmula de variación considerando las cartas distinguibles;

- C3: Suponer que se pueden repetir elementos, que aparece en el $24 \%$ de los estudiantes sin instrucción de Navarro Pelayo.

- C4: Interpretación incorrecta de términos matemáticos. Uno de nuestros estudiantes confunde la palabra posibilidad y la interpreta como probabilidad, aplicando la regla de Laplace para resolver el problema 1.

Otros conflictos se producen en el desarrollo del procedimiento en estudiantes que, 
aparentemente han comprendido el enunciado, pero fallan al aplicar algún tipo de estrategia:

- Enumeración incorrecta. Muchos estudiantes plantean la enumeración de unos pocos casos, y al tratar de generalizar, no lo hacen correctamente, por lo que cuentan combinaciones en exceso o defecto; por ejemplo, en la Figura 2 se mostró la respuesta de JG, quien aplica una estrategia de enumeración sistemática, pero incompleta, o AM quien realiza la enumeración excesiva (Figura 9). En la investigación de NavarroPelayo el 60,4\% de estudiantes sin instrucción realiza enumeraciones incorrectas.

- Construcción incorrecta de tablas. De los 11 estudiantes que tratan de construir una para resolver el Problema 2, sólo 2 construyen una tabla que corresponda al enunciado y les facilite la posterior enumeración. El resto falló en la construcción, lo que les llevó a usar el principio multiplicativo en forma incorrecta.

- No se llega a reconocer la operación combinatoria de combinación en el enunciado, por lo que no aplican la fórmula correspondiente, que también se identifica en Navarro-Pelayo.

- Construcción incorrecta del diagrama en árbol. Algunos estudiantes que tratan de construir un diagrama en árbol como ayuda a la enumeración, no llegan a completarlo, o se equivocan en el número de nudos o ramas, con lo cual llegan a soluciones incorrectas del problema.

Finalmente citamos casos aislados de generalización incorrecta o división incorrecta del problema en partes en los estudiantes que aplican estas estrategias.

\section{Conclusiones}

Los resultados muestran escasa capacidad de razonamiento combinatorio de los futuros profesores que participan en nuestro estudio al resolver los problemas planteados, que fueron los más sencillos en las investigaciones de Navarro-Pelayo (1994) y Roa (2000).

Se muestra poca competencia en el uso del lenguaje matemático, pues el utilizado es eminentemente verbal que fue el único empleado en algunas respuestas. Muy pocos estudiantes utilizan el diagrama del árbol, a pesar del carácter de útil de resolución que le otorga Fischbein (1975) y cuando se usa está incompleto o mal construido, llevando a una respuesta incorrecta. El lenguaje icónico fue utilizado para simbolizar los elementos que intervienen en la situación planteada, como por ejemplo dibujar las cartas y los sobres en el 
Problema 1, o los cromos en el problema 3. El simbólico se implementó para asociar, ya sea con números o letras, los elementos involucrados en la situación planteada.

Las estrategias aplicadas son en su mayoría incorrectas o parcialmente correctas, debido a que las enumeraciones no son completas o bien no son sistemáticas, incluso aunque el número de elementos a combinar es pequeño. Aunque la enumeración sistemática o la fijación de variable, si son bien aplicadas otorgan resultados correctos, las dificultades se encontraron en la generalización de los procesos parciales de enumeración. Los pocos participantes que usan fórmulas las plantean incorrectamente, lo que también sucede al emplear el principio multiplicativo. Es muy escaso el uso de estrategias generales de resolución de problemas, que podrían aplicarse en los problemas combinatorios con éxito, según Roa (2000).

Finalmente destacamos la presencia de conflictos semióticos en los futuros profesores en la interpretación del enunciado y el desarrollo de la solución, que fueron interpretados como errores por Navarro-Pelayo y nosotros atribuimos a fallos en procesos interpretativos.

Estos resultados muestran, por un lado, la complejidad semiótica de los problemas combinatorios, incluso tan sencillos como los planteados en esta investigación, debido a los múltiples procesos interpretativos que se requieren, por un lado, para comprender el enunciado y por otro a la necesidad de dominio de estrategias para la resolución del problema.

Los participantes han confundido el tipo de objetos a combinar (distinguibles o no), la existencia o no de orden, la posibilidad o no de repetir elementos y han confundido términos matemáticos, como posibilidad, debido al diferente uso de que de ellos se hace en la vida diaria y la matemática. Un desafío en la enseñanza sería alcanzar una buena conexión entre lenguaje de cada día y lenguaje matemático, pues los términos matemáticos tienen mayor precisión que el lenguaje ordinario, ya que proporcionan definiciones necesarias y suficientes, mientras que el lenguaje ordinario es simplemente descriptivo (Schleppegrell, 2007).

La enseñanza de la combinatoria elemental se debe potenciar no sólo en el aula, sino también en la formación de profesores, debido a la utilidad de la misma en el aprendizaje de resolución de problemas, que es un bloque transversal del currículo desde los primeros cursos de la Educación Primaria. Es por ello también necesario continuar la evaluación del razonamiento combinatorio de los futuros profesores, continuando la investigación presentada en este trabajo. 


\section{REFERENCIAS}

BATANERO, Carmen; GODINO, Juan Díaz; NAVARRO-PELAYO, Virginia (1994). Razonamiento combinatorio. Madrid: Síntesis.

BATANERO, Carmen; NAVARRO-PELAYO, Virginia; GODINO, Juan Díaz. Effect of the implicit combinatorial model on combinatorial reasoning in secondary school pupils.

Educational Studies in Mathematics, v. 32, n.1, p. 181-199. 1997.

DUBOIS, Jean. Une systématique des configurations combinatoires simples. Educational Studies in Mathematics, v. 15, n.1, p. 37-57. 1984.

DUVAL, Raymond. Comment analyser le fonctionnement représentationnel des tableaux et leur diversité?. Spirale-Revue de recherches en éducation, v. 32, n. 32, p. 7-31. 20031

ENGLISH, Lynn. Assessing for structural understanding in children's combinatorial problem solving. Focus on Learning Problems in Mathematics, v. 21, n. 4, p. 63-82. 1999,

ENGLISH, Lynn. Combinatorics and the development of children's combinatorial reasoning. En: JONES, Graham (Ed.), Exploring probability in school. Boston, MA: Springer. 2005, p. 121-141.

FISCHBEIN, Efraim. The intuitive sources of probabilistic thinking in children.

Dordrecht: Reidel. 1975.

GABUCIO, Fernando; MARTÍ, Eduardo; ENFEDAQUE, Jesús; GILABERT, Sandra: KONSTANTINIDOU, Aikaterini. Niveles de comprensión de las tablas en alumnos de primaria y secundaria. Cultura y Educación, v. 22, n.2, p. 183-197. 2010.

GODINO, Juan Díaz. Un enfoque ontológico y semiótico de la cognición matemática. Recherches en Didactique des Mathématiques, v. 22 n. 2-3, p. 237-284. 2002.

GODINO, Juan Díaz; BATANERO, Carmen; Font, Vincenç. The onto-semiotic approach to research in mathematics education. ZDM. The International Journal on Mathematics Education, v. 39 n. 1-2, p. 127-135. 2007.

GODINO, Juan Díaz; BATANERO, Carmen; Font, Vincenç. The onto-semiotic approach: implications for the prescriptive character of didactics. For the Learning of Mathematics, v.39, n.1, p. 38-43. 2019.

GODINO, Juan Díaz; BATANERO, Carmen; ROA, Rafael. An onto-semiotic analysis of combinatorial problems and the solving processes by university students. Educational Studies in Mathematics, v. 60, n.1, p.3-36. 2005.

GRAVEMEIJER, Koeno; LEHRER, Richard; VAN OERS, Bert; VERSCHAFFEL, Lieven. Symbolizing, modeling and tool use in mathematics education. New York; Springer Science \& Business Media. 2013. 
INHELDER, Brigitte; PIAGET, Jean. The growth of logical thinking: from childhood to adolescence. London: Routledge and Kegan Paul.1958.

JALAN, Sukoriyanto; NUSANTARA, Toto; SUBANJI, Subanji; CHANDRA, Tjang. Students' thinking process in solving combination problems considered from assimilation and accommodation framework. Educational Research and Reviews, v.11, n.16, p. 1494-1499. 2016.

LOCKWOOD, Elise. A model of students' combinatorial thinking. The Journal of Mathematical Behavior, v. 32, n. 2, p. 251-265. 2013.

\section{MECD, MINISTERIO DE EDUCACIÓN, CULTURA Y DEPORTE. Real Decreto} 1105/2014, de 26 de diciembre por el que se establece el currículo básico de la Educación Secundaria Obligatoria y del Bachillerato. Madrid: MECD, 2015.

MELUSOVA, Janka; VIDERMANOVA, Kitti. Upper-secondary students' strategies for solving combinatorial problems. Procedia-Social and Behavioral Sciences, v. 197, n, 1, p. 1703-1709. 2015.

NAVARRO-PELAYO, Virginia. Estructura de los problemas combinatorios simples y del razonamiento combinatorio en alumnos de secundaria. Tesis Doctoral (Doctorado en Didáctica de la Matemática). Universidad de Granada. Granada, España, 1994. Orientadores: Carmen Batanero y Juan Díaz-Godino.

PESSOA, Cristiane; BORBA, Rute. O desenvolvimento do raciocínio combinatório na escolarização básica. Em Teia| Revista de Educação Matemática e Tecnológica Iberoamericana, v, 1, n, 1, p. 1-22. 2010.

PESSOA, Cristiane; BORBA, Rute Quem dança com quem: o desenvolvimento do raciocínio combinatório de crianças de $1^{\mathrm{a}}$ a $4^{\mathrm{a}}$ série. Zetetike, v. 17, n.1, p. 105-149. 2009.

ROA, Rafael. Razonamiento combinatorio en estudiantes con preparación matemática avanzada. Tesis Doctoral (Doctorado en Didáctica de la Matemática). Universidad de Granada. Granada, España, 2000. Orientadores: Carmen Batanero y Juan Díaz-Godino.

ROA, Rafael; BATANERO, Carmen; GODINO, Juan Díaz; CAÑIZARES, María Jesús. Estrategias de resolución de problemas combinatorios por estudiantes con preparación matemática avanzada. Epsilon, v, 36, n. 1, 0. 433-446. 1997.

SCHLEPPEGRELL, M. (2007). The linguistic challenges of mathematics teaching and learning: A research review. Reading and Writing Quarterly, v. 23, n.1, p. 139-159.

\section{Agradecimientos:}

Proyecto EDU2013-41141-P (AEI-FEDER) y grupo FQM126 (Junta de Andalucía). 


\section{$\underline{\text { SOBRE OS AUTORES }}$}

\section{María M. Gea}

Doctora en Educación, Universidad de Granada, España (UGR); Profesora de Didáctica de la Matemática. Universidad de Granada. Grupo de Investigación en Educación Estadística de la Universidad de Granada. E-mail: mmgea@ugr.es

ORCID http://orcid.org/0000-0002-5229-0121

\section{Carmen Batanero}

Doctora en Matemáticas, Universidad de Granada, España (UGR). Catedrática de Didáctica de la Matemática. Universidad de Granada. Grupo de Investigación en Educación Estadística de la Universidad de Granada. E-mail: batanero@ugr.es

ORCID http://orcid.org/0000-00024189-7139

\section{Alex Venegas}

Máster en Didáctica de las Matemáticas, Universidad de Granada, España (UGR). Profesor de Estado en Matemática y Computación en el Colegio Santa María Eufrasia, Chile. Beca CONICYT PFCHA 77170016. E-mail: a.venegas.guerrero@gmail.com

ORCID http://orcid.org/0000-0002-9505-5383 